\title{
A Novel Structure to Enhance Second Harmonic Generation in Plasmonic Waveguide using Optimized Plasmon Polariton Nanoparticles
}

Mohammadreza Zarei ( $\square$ jaliani1363@gmail.com )

Islamic Azad University of Fasa https://orcid.org/0000-0003-3627-4217

Abbas Kamali

Islamic Azad University of Fasa

Mohsen Imanieh

Islamic Azad University of Fasa

Ali Emam ghorashi

Islamic Azad University of Fasa

\section{Research Article}

Keywords: SHG, MGIM, plasmonics, Graphene, grating, asymmetric structure

Posted Date: May 19th, 2021

DOI: https://doi.org/10.21203/rs.3.rs-485633/v1

License: (a) (i) This work is licensed under a Creative Commons Attribution 4.0 International License.

Read Full License 


\title{
A Novel structure to enhance second harmonic generation in plasmonic waveguide using optimized plasmon polariton nanoparticles
}

\author{
Mohammadreza Zarei ${ }^{1}, \quad$ Abbas Kamali ${ }^{2}, \quad$ Mohsen Imanieh ${ }^{3}$, Ali Emam ghorashi ${ }^{4}$ \\ Department of Electrical Engineering, Fasa Branch, Islamic Azad University, Fasa, Iran \\ 1. Corresponding Author: jaliani1363@ gmail.com, phone number: 53331838 - 071 \\ 2. abbas_kamaly@yahoo.com, phone number: 53331838 - 071 \\ 3. m_imanieh@yahoo.com, phone number: 53331838 - 071 \\ 4. aemamghorashi@iaufasa.ac.ir, phone number: 53331838 - 071
}

\begin{abstract}
In this paper, we propose a new structure for the plasmonic waveguide to optimize the nonlinear optical effect of the second harmonic generation (SHG). For this purpose, we use a layer of graphene grating and spherical and elliptical plasmon polariton nanoparticles made of gold and silver. The number, location and material of nanoparticles as well as the number of graphene layer gratings are optimized to achieve the highest possible nonlinear effect. The simulation results
\end{abstract}

\section{INTRODUCTION}

With the approach of technology to the integration of optical electronic circuits, construction problems and phenomena that helped prevent further compression of the structure, has led to the use of plasmonic structures and plasmonic waves to be studied and used by researchers. These nanostructures are composed of metal and dielectric and their dimensions are below the excitation wavelength, i.e. the wavelength of the beam that excites the plasmonic waves [1]. Plasmonics is based on the process of interaction of electromagnetic waves and conducting electrons in nanoscale metals. Using nanoscale (or plasmonic) optics, one can step beyond the limits of light. In nano-plasmonics, researchers focus rays at the nanoscale by converting free photons into stabilized charge fluctuations, known as surface plasmons. These plasmons act as a radio antenna. Although many advances have been made in the design of terahertz transmitters, plasmonics have physical limitations due to their high absorption by various materials and the consequent reduction in their intensity. The biggest obstacle to the use of plasmonics is attenuation in the range of visible light wavelengths [8-2].

Around 1956, Pence analytically explained the rapid decline in the energy of electrons in the passage of metals. The reason for this naming was the similarity of these electron oscillations with the oscillations of the particles in the plasma showed that the optical power of SHF wave and peak optical power of SHF is increased noticeably using grating layer of graphene along with optimized plasmon polariton nanoparticles.

Keywords: SHG, MGIM, plasmonics, Graphene, grating, asymmetric structure

environment. In the same year, Fano used the term polariton to oscillate metal-bound electrons when coupled to radiant photons [13]. The name polariton is used for quasi-particles that were half-particle and half-photon, which is the state coupled between a photon, the primary excitation beam, and the metal-conducting electrons. .

Polaritons are surface plasmons (SPPs) electromagnetic excitations that are coupled to electrons that collect and oscillate freely in metals so that they can propagate longitudinally at the metal-dielectric junction.

The important reason for the polarity of surface plasmons is their ability to focus the electromagnetic field, which reduces the diffraction limit at nanometer optical waves and improves the expansion of localized fields by several large magnitudes. This causes the polarity emission modes of surface plasmons, ie the transverse electric field (TE) or the transverse magnetic field (TM), to be confined to the planes perpendicular to the direction of propagation.

Exactly at the plasmon frequency, $\omega p$, the electron gas intensifies and fluctuates sharply. This resonant frequency increases with the electron density, n. Similarly, the force constant of an electric return spring is directly proportional to the charge displacement. In fact, it can be shown that the plasmon frequency is directly related to the square root of the electron density. An electric field cannot exist inside a metal because the electrons of the metal interact with the electric field and create an opposite field called the plate field. An example of this is the image load, which neutralizes the field 
each external load. This is also true for an electromagnetic wave. That is, electrons respond to changes in the external field and resist it at any moment. As a result, the electromagnetic wave cannot enter the metal and is therefore reflected back. But at high frequencies we get to the point where the external field oscillates very quickly and the electrons cannot follow those oscillations. At frequencies beyond this frequency, a metal loses its reflectivity. The energy corresponding to this energy is called plasmon energy, it is equivalent to $E=\eta \omega_{p}$ and its value is about 10.30 electron volts. This property can be used to build energy storage windows so that it passes visible light but reflects heat radiation and heat is not wasted. We know that single electron waves are quantized when enclosed in a nanostructure. Similarly, batch waves of electrons, or plasmons, are affected by the boundary conditions of a thin film, a nanorod, or a nanoparticle.

Various plasmonic waveguides, like insulator-metal-insulator (IMI), Metal-Graphene-insulator-Metal (MGIM), channel plasmon polaritons (CPP), dielectric-loaded SPP wave guides (DLSPPW) and hybrid plasmonic waveguide, are projected in theory and by experimentation [9-12]. Among these, the MGIM structure is especially engaging thanks to its glorious glorious, acceptable SPP propagation length, simple fabrication, and efficient couplings with optical fiber and $\mathrm{Si}$ waveguides [7, 8]. On the opposite hand, MGIM waveguides have higher confinement factors and nearer spacing to adjacent waveguides $[13,14]$ and are projected for tons of applications like Optical resonators [15-17], wave guide bends and splitters supported MGIM sub-wavelength plasmonic waveguides [1820] and resonator [21, 22]. Surface plasmon polariton waves in metal-insulator-metal hierarchic grating wave guide for caparison of SPP waves also are investigated [23, 24].

The most MGIM waveguides studied antecedental have bilaterally symmetric structure, that means that the metals at the highest and bottom of the stuff layer are an equivalent. Also, uneven MGIMs haven't been investigated, and to the simplest of our data, many researchers have investigated the non-linear optical phenomena in these uneven waveguides. Moreover, very little attention has thus far been paid to the SHG in plasmonic waveguides, and this issue on MGIM plasmonic waveguides is nevertheless to be studied extensively. Among the nonlinear processes, SHG is often studied due to its fascinating applications and easy theoretical principle [25]. SHG is an efficient thanks to extend optical device wavelengths to visible or ultraviolet [26]. However, introducing sturdy nonlinear responses from materials to understand to understand within the sub-wavelength scale continues to be difficult.

A number of reports on SHG sweetening in plasmonic nanostructures are printed. Simon et al. first rumoured Associate in Nursing experimental and theoretical investigation on the coupling of SHG light-weight to surface plasmons in skinny silver films and ascertained harmonic sweetening to 1 and a [*fr1] orders of magnitude thanks to excitation of surface plasmons [25]. SHG sweetening with metal tips, homogenised centro-symmetric nanowires, hybrid plasmonic waveguides, bowtie aperture arrays, plasmonic slot waveguides has additionally been investigated [28 - 32].

In Silicon, however, SHG can't be directly excited. the explanation is that the second-order susceptible vanishes during this material, as a result of the crystal centro-symmetry. Exploitation of Si compound (Si3N4) has been projected to unravel this disadvantage and induces second-order nonlinear processes in Si compatible structures [33-36]. a lot of recently, Oliveira et al. rumoured SHG in a very twenty $\mu \mathrm{m}$ radius Si3N4 ring resonator by exploitation the electric-field evoked SHG method and calculated a conversion potency of concerning three. $68 \times 10-3$ with a pumping power of $75 \mathrm{mw}$ [35]. so as to more increase the potency of SHG and cut back the sizes of devices, the LiNbO3 crystal in plasmonic based mostly nonlinear devices ar among the foremost promising candidates to match this expectation, as a result of their ability to permit sturdy native increased confinement of sunshine on the far side the bounds obligatory by the laws of optical phenomenon in stuff media [37]. many types of plasmonic structures are projected. for example, economical SHG has been conferred in plasmonic slot waveguides (PSW) [26], long-range plasmonic waveguides [38], hybrid plasmonic waveguides (HPW) [39], metal surfaces with nano-scale roughness [40], individual aluminiferous nano-aperture [39], plasmonic particle chains [40], and plasmonic core-shell nanowires [41].

Lithium niobate may be a prolific optoelectronic material. It continues to be used in devices starting from surface acoustics wave (SAW) filters and modulators in physics to electro-optic modulators, q-switches and frequency conversion in optics. What makes metallic element niobate such a pretty material platform is its massive electricity, electro-optic and nonlinear optical properties. The nonlinear optical properties of metallic element niobate have additionally created it Associate in Nursing more and more in style material for optical frequency conversion. metallic element niobate has comparatively massive quadratic susceptibilities. That is, the polarisation evoked within the crystal that's proportional to the merchandise of 2 incident field elements is substantial, particularly at optical high powers $[42,43]$. From our data, the foremost used nonlinear material in these structures to understand SHG is metallic element mineral [25]. However, in spite of the commonly used continuous wave $(\mathrm{CW})$ pump power of the elemental frequency $(\mathrm{FF})$ to concerning one $\mathrm{W}$, the height powers of the generated second harmonic frequency (SHF) ar typically restricted to $10-5 \mathrm{~W}$ [26]. albeit it is exaggerated to $10^{-2} \mathrm{~W}$ in $\mathrm{HPW}$, the corresponding wave guide length to understand this potency is one millimetre [27], that is maybe too long and not appropriate for application in future integrated nano-photonic circuits. The rather tiny rumoured efficiencies are thanks to the comparatively tiny nonlinear susceptible in crystal, the moderately massive nonlinear coupling coefficients between completely different frequencies, and also the absorption loss of the plasmonic modes.

In this paper, we investigate the nonlinear optical effect of the second harmonic production on plasmonic waveguides of metal, insulation and metal, and propose a new structure for 
plasmonic waveguides that, in addition to having a high nonlinear effect, also have a high propagation length. The proposed structure has a graphene grate as well as a combination of polariton plasmon nanoparticles in spherical and elliptical shapes. The number, location and material of nanoparticles as well as the number of graphene layer gratings are optimized to achieve the highest possible nonlinear light effect.

This paper is organized as follows. In Section two we have a tendency to describe the structure of the proposed waveguide. The theory of proposed nanoparticles and optimization method is presented in this section as well. In Section three, the simulation results of SHG within the proposed structure are conferred. In Section four, the paper is summarised.

\section{THE STRUCTURE OF MGIM WAVEGUIDE}

Figure 1 shows the grating graphene MIM plasmonic waveguide structure into consideration. As shown in the figure, this structure consists of two layers of metal at the top and bottom of the structure and a crystal layer of lithium niobytes (LiNbO3). A grating graphene layer is also added between the top metal and the crystal layer. The grate graphene layer leads to more absorption of photons and increases the absorption coefficient in the middle layer, thus increasing the production of the second harmonic. In addition, a number of spherical and elliptical nanoparticles of gold or silver have been added in the center of the layer. As will be shown, these nanoparticles increase the intensity of the optical field and thus increase the second harmonic effect. The number, shape (spherical or elliptical), and the material (gold or silver) of nanoparticle would be optimized to reach the maximum possible of SHG.

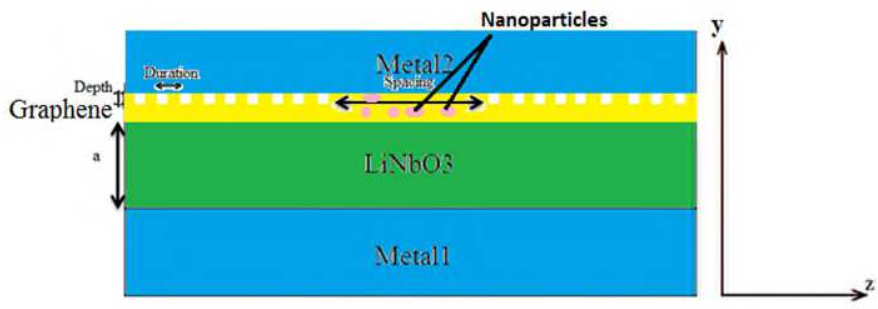

Figure 1. Structure of the proposed GRATING GRAPHENE MGIM waveguide under consideration

For optimizing the SHG of the structure, nano-particles are used and the number and the shape is optimized using coupled-dipole approximation (CDA) method. The CDA method is a powerful tool for simulating nanoparticles with intermittent structures. The schematic of an alternating and non-alternating structure is shown in Figure 2. In this figure, all the nanoparticles are at the same distance from the side nanoparticles. This figure shows one quarter of the center of graphene layer for simplicity of optimization. Since the adsorption of nanoparticles is highly dependent on the arrangement of nanoparticles, it is expected that intermittent adsorbents will have better adsorption. A major advantage of this method is its use when using evolutionary optimization algorithms.

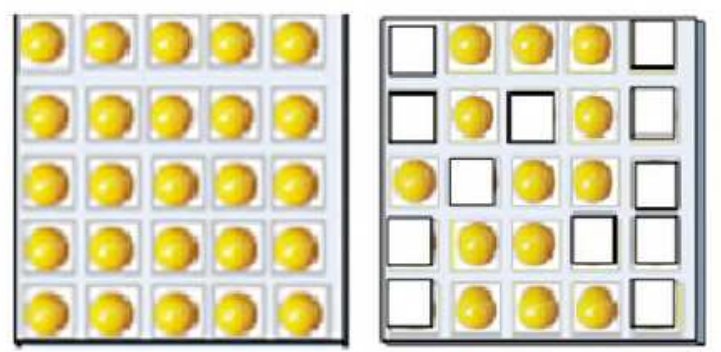

Figure 2. an alternating and non-alternating structure of nano-particles.

In the first step, the position of each nanoparticle in the direction of $\mathrm{x}, \mathrm{y}, \mathrm{z}$ should be calculated. The nanoparticle positioning algorithm is shown in Figure 3.

For $i=1: N 1$
For $j=1: N 2$
For $k=1: N 3$
If $B(i, j, k)==1$
$r=[r[(i-(N 1+1) / 2) * d ;(j-(N 2+1) / 2) * d ;(k \quad(N 3+1) / 2) * d] \cdot 1] ;$
End; End; End; End;

Figure 3. Nanoparticle positioning algorithm

In this quasi-code $\mathrm{r}$, the position of the nanoparticles and $\mathrm{N} 1$, $\mathrm{N} 2, \mathrm{~N} 3$ is also the number of nanoparticles in the direction of $\mathrm{I}, \mathrm{j}, \mathrm{k} . \mathrm{D}$ is the distance between the nanoparticles and the vector $B(i, j, k)$ is a three-dimensional representation of binary numbers. If a particle of this matrix becomes one, it means the presence of a particle in position $\mathrm{i}, \mathrm{j}, \mathrm{k}$, and if a particle becomes zero, it means that the particle is not present in position $\mathrm{i}, \mathrm{j}, \mathrm{k}$.

In the second step, we need to model the effect of the input field on the nanoparticles. To do this, we assume that a plane field is entered on the desired plane as:

$\mathbf{E}_{i n c}(\mathbf{r}, t)=\mathbf{E}_{0} e^{j\left(\mathbf{k r}_{s}-\omega t\right)}$

If the incoming light is entered at an angle of $\theta, \varphi$ the field in each nanoparticle can be considered as follows: 


$$
\mathbf{E}_{i n c}\left(\mathbf{r}_{s}\right)=\mathbf{E}_{0} e^{j \mathbf{k} \cdot \mathbf{r}_{\mathrm{s}}}
$$

In these relations, the parameters $\lambda \mathrm{r}, \mathrm{t}, \omega, \mathrm{k}=\omega / \mathrm{c}=2 \pi / \lambda$, $\mathrm{c}$, respectively, are the wavelength of the incoming light, the speed of light, the wave vector, the angular frequency, the time and the position vector. In this regard, the parameter $\mathrm{k}$ is also calculated from the following equation:

$\mathbf{k}=\frac{2 \pi}{\lambda} \hat{k}=\frac{2 \pi}{\lambda}[\sin (\theta) \cos (\varphi), \sin (\theta) \sin (\varphi), \cos (\theta)]$

For the input field with polarization P, the intensity of E0 field is equal to:

$$
\mathbf{E}_{0}=\left[\sin \left(\theta-\frac{\pi}{2}\right) \cdot \cos (\varphi), \sin \left(\theta-\frac{\pi}{2}\right) \cdot \sin (\varphi), \cos \left(\theta-\frac{\pi}{2}\right)\right]
$$

We also have $\mathrm{S}$ for polarization field:

$$
\mathbf{E}_{0}=\left[\cos \left(\phi+\frac{\pi}{2}\right), \sin \left(\phi+\frac{\pi}{2}\right), 0\right]
$$

When a field is applied to a particle, the polarization coefficient $\alpha$ is equal to:

$$
\alpha_{s}=V \varepsilon_{0} \frac{\varepsilon_{r}-1}{1+L_{1}\left(\varepsilon_{r}-1\right)}
$$

In this regard, $\varepsilon r=\varepsilon_{\text {particle }} / \varepsilon_{\text {medium, }} \varepsilon 0$ is the coefficient of passage of free space. Also, $\mathrm{V}$ is the nanoparticle volume and L1 is the shape factor. For spherical nanoparticles, the shape coefficient is calculated as follows according to the values of $\mathrm{a}, \mathrm{b}, \mathrm{c}$ :

$$
\begin{aligned}
e^{2} & =1-\frac{b^{2}}{a^{2}} \quad, f^{2}=\frac{b^{2}}{a^{2}}-1 \\
L_{1} & =\frac{1+f^{2}}{f^{2}}\left[1-\frac{1}{f} \tan ^{-1}(f)\right] \quad \text { oblate }(a<b) \\
L_{1} & =\frac{1-e^{2}}{e^{2}}\left(-1+\frac{1}{2 e} \ln \frac{1+e}{1-e}\right) \quad \text { prolate }(a>b)
\end{aligned}
$$

Figure 4 shows the schematic of nanoparticles with different values of $a, b, c$.
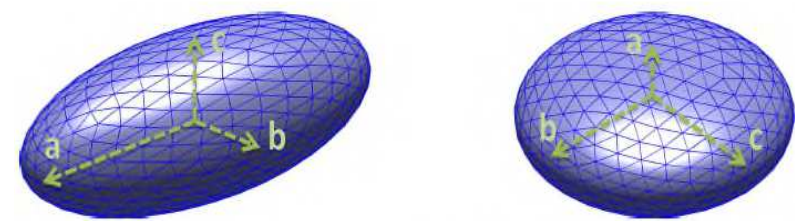

Figure 4 nanoparticles with different values of a, b, c

Now we can define the electric bipolar vector for each nanoparticle as follows:

$$
\mathbf{P}_{s}=\varepsilon_{0} \alpha_{s} \mathbf{E}_{L o c}\left(\mathbf{r}_{s}\right)
$$

In this connection, $\mathrm{P}$ is the induced dipole moment and $\mathrm{E}$ is the electric field of each nanoparticle. Therefore, the field within each nanoparticle depends on two factors. An input field. The second field is caused by the radiation of other nanoparticles.
The sum of these two field fields makes up each nanoparticle, which is calculated from the following equation:[9]

$$
\mathbf{E}_{l o c, s}=\mathbf{E}_{i n c, s}+\mathbf{E}_{d i p, s}=\mathbf{E}_{0} e^{j \mathbf{k} \mathbf{r}_{s}}-\sum_{s \neq h} \mathbf{A}_{s, h} \mathbf{P}_{s}
$$

In this respect, $\mathrm{Ps}$ is the same as the dipole moment induced in s. Also, As, h is a $3 \times 3$ matrix that is calculated as follows.

$$
\left\{\begin{array}{l}
\mathbf{A}_{s, h}=\frac{\exp \left(j \mathbf{k} \mathbf{r}_{s, h}\right)}{\mathbf{r}_{s, h}}\left[k^{2}\left(r_{s h} r_{s h}-\mathbf{I}_{3 \times 3}\right)+\frac{j k \mathbf{r}_{s h}-1}{\mathbf{r}_{s h}^{2}}\left(3 r_{s h} r_{s h}-\mathbf{I}_{3 \times 3}\right)\right] s \neq h \\
\mathbf{A}_{s, s}=\frac{1}{\alpha_{s} \varepsilon_{0} \mathbf{I}_{3 \times 3}}
\end{array}\right.
$$

In the third step, the equation must be solved and the vector $\mathrm{P}$ calculated from it. In this case, A is a $3 \mathrm{~N} \times 3 \mathrm{~N}$ tensor.

\section{Results and discussion}

Figure 5 shows four SHG diagram with different numbers of nanoparticles.
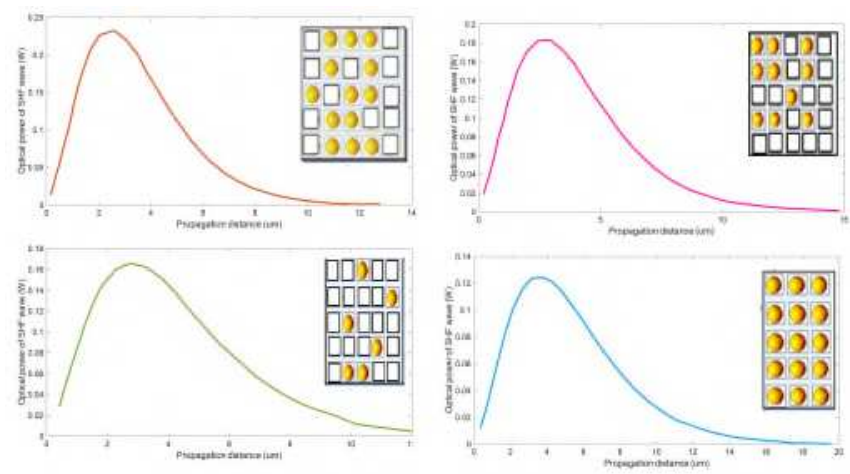

Figure 5 Comparison of SHG for periodic and nonperiodic structures

Figure 5 shows the second harmonic optical power as a function of the propagation length for different structures for nanoparticles (different location, number and shape). As it is known, with the change of location and number of nanoparticles, the maximum optical power of the second harmonic and the propagation length of the second harmonic change drastically. The maximum second harmonic power is 0.23 watts, which is almost 6 times the output power of ordinary plasmonic waveguides. This structure also has the shortest emission length of 2.4 micrometers. Due to the production of the second harmonic along the waveguide as well as its attenuation along the same waveguide, there is always a point in the propagation length at which the second harmonic reaches its maximum value, which is called the optimal propagation length. Due to the fact that the dimensions of optical integrated circuits are very small, the optimal propagation length may be larger than the dimensions of the 
circuit and we cannot reach the second maximum harmonic in the desired circuit. The smaller the optimal propagation length, the better.

The nanoparticle structure can be optimized to achieve a higher second harmonic optical power and shorter propagation length. Since for a $5 \times 5$ array the number of possible states is $2^{25}=33554432$, not all possible states can be considered. That's why we're looking at binary optimization algorithms. One of the most popular binary optimization algorithms is the bird algorithm. Below is a summary of binary bird algorithms.

Particle Optimization Algorithm is a search algorithm based on social exchanges and behaviors that is modeled on the group behavior of weight class birds. This algorithm is an evolutionary algorithm that was first developed in 1995 by Kennedy and Heart. The bird algorithm, like other evolutionary algorithms without the need for derivation, is population-based and random. This algorithm is a mass-based algorithm that simulates the behavior of a group of birds (a group of particles) that, in search of a location, sampling different points and pushing the group toward a more hopeful point (points where the answer is likely to be reached). There are more nearby). The algorithm collects the information collected by the particles inside the batch. A particle, according to the path of motion defined by its velocity, moves towards the response space (search). The velocity of each particle is determined by the personal experiences of the particle and the experiences of its neighboring particles. Therefore, during the search process, the particles tend to move toward better points in the response space.

In this algorithm, one particle consists of five components:

1- X: A vector that shows the current location of this particle in the search space (answer). The size of $\mathrm{x}$ is determined by the number of variables used by the problem 2 - Fit: A numeric value that indicates the proximity of the vector $\mathrm{x}$ in the search space to the optimal point. In fact, the amount of gameplay determines the quality of this vector. This number is calculated according to the specific evaluation function (fit) for each problem.

3-: A vector, which gives the velocity of each particle. This velocity indicates the rate of change related to the vector $\mathrm{x}$ in the next iteration. Manipulating the vector value causes a slight change in the motion of this particle in the search space

4- Personal fitness $\left({ }^{\text {fittness }_{p}}\right)$ : The best amount of fitness that a particle has started from the beginning until now.

5. Pbest (personal best): A copy of the $\mathrm{x}$-bearer is the best answer a bit has ever received. This vector fits fittness $_{p}$. 6- In addition, every seed is aware of the (global best) gbest, which is the best pbest in the neighborhood of all the particles and all repetitions. A neighborhood may consist of a small group of seeds or may contain all the particles present. Group fit ( fittness $_{g}$ ) the fit value is proportional to gbest.

The core of the particle optimization algorithm is actually the process by which the velocity vector (v) changes. By applying this change, the components of the more promising parts search the answer space many times. At each stage, the velocity of each vowel changes in such a way that the movement towards the pbest and gbest takes place. Moving to these two locations are weighted by different random quantities. This weighting accelerates this movement. These random values cause the size of each step to change with time. This causes the algorithm to be caught in non-optimal and local responses. Each new speed is calculated according to the current speed and the current distance from. The $\mathrm{i}$ and $\mathrm{d}$ indices represent the particle number index and the index number (axis) of that particle, respectively. Also, the new location of each field is obtained according to the current location and the new speed.

$V_{i_{-} d}^{k+1}=w V_{i_{-} d}^{k}+c_{1} r_{1}\left(\right.$ pbest $\left._{i_{-} d}-x_{i_{-} d}^{k}\right)+c_{2} r_{2}\left(\right.$ gbest $\left._{d}-x_{i_{-} d}^{k}\right)$

$\underset{\substack{i \_d \\ x_{i \_d}^{k}}}{x_{i-d}^{k+1}}=x_{i-d}^{k}+V_{i_{-} d}^{k+1}$

Shows the current location of the i-particle in the repetition of $\mathrm{k}$ and the center of d. ${ }^{V_{i \_d}}$ also indicates the velocity of the iM particle in the $\mathrm{km}$ repetition. $\mathrm{w}$ is the criterion for the inertial weight of motion, $\mathrm{c} 1$ and $\mathrm{c} 2$ are the coefficients of constant acceleration and $\mathrm{r} 1$ and $\mathrm{r} 2$ are equal to the values between zero and one. As can be seen in the above equations, there are three parts for calculating velocity changes. The first part, which is referred to as the size of the movement, indicates the effect of the previous direction and speed on the new speed $\left(w V^{k}{ }_{i \_} d\right)$. In other words, this section shows the tendency of the particle to move in the previous direction. Each repetition of the previous value $\mathrm{v}$ determines the magnitude of the motion of that particle in the next repetition. This characteristic causes the particles to escape from the local optimal values. The second part is characterized as individual learning, moving the movement towards the best response ever found. This section shows the experiences of the particle itself. The third part, called social learning, leads the particle to the best answer ever found by all particles. This part is actually the experience of the group that each particle uses.

The position of the particles in the binary model is determined by two values of zero or one in each dimension. Using the same relation as before to change the velocity of each particle is used without change, and of course with the difference that the velocity as a defined probability must be converted to the interval (0 and 1). A logistics conversion function is used for this purpose.

$\operatorname{sigmoid}\left(v_{i d}^{k}\right)=\frac{1}{1+e^{\left(-v_{i d}^{k}\right)}}$

We also use the following relationship to update the position of the birds:

$x_{i d}^{k}=\left\{\begin{array}{cc}1, & \text { if rand }<\operatorname{sigmid}\left(v_{i d}^{k}\right) \\ 0 & \text { otherwise }\end{array}\right.$

Here, in order to optimize the optical power and propagation length, the binary bird algorithm must select a sequence from the existing sequences that has the better optical plwer. In other words, the binary algorithm of birds controls the 
presence (or absence) of nanoparticles in a particular situation by producing the optimal binary matrix. As mentioned above, the bird algorithm is an algorithm that minimizes the function of the target. To use the binary bird algorithm, the objective function must be defined as follows:

Cost Function $=+\sum_{i=1200}^{1650} P_{S H G}$

Cost Function=- $\sum_{i=1300}^{1650} L_{S H G}$

In these relations, $i$ is the same wavelength and $\mathrm{P}_{\mathrm{SHG}}$ and $\mathrm{L}_{\mathrm{SHG}}$ are the optical power and propagation length of SHG, respectively. Here, a negative sign causes the bird algorithm to minimize the target function. The bird algorithm was applied to the target function of optical power and propagation length of SHG and the result of Figure 6 is obtained. The optimized nanoparticle structure is shown in this figure as well.

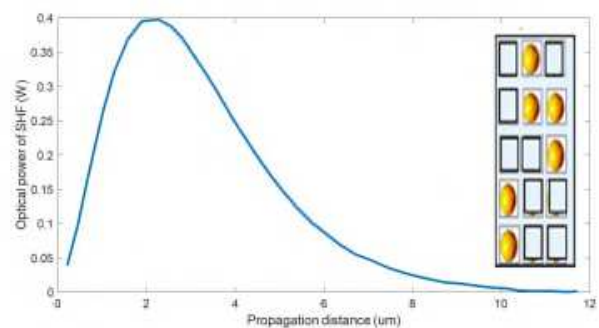

Figure 6. the optical power of SHG for proposed structure with optimized nanoparticles

In the next step, we will optimize the thickness and spacing of the graphene grating layer. Figure 7 shows the optical power of the SHF peak and the position of the SHG process peak relative to the grating spacing for a $1 \mathrm{~W}$ pump power. It can be seen that there is an optimal point for the spacing that is the maximum optical power of the SHF peak and the minimum peak position. For a spacing of $1.3 \mathrm{um}$, the optical power of the SHF peak is $0.44 \mathrm{~W}$ and the corresponding position is 1.2 um. So with two different nanoparticles and the use of a grating layer of graphene, SHG is greatly improved.

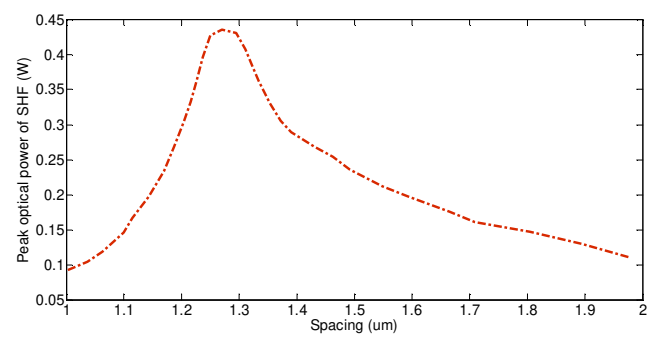

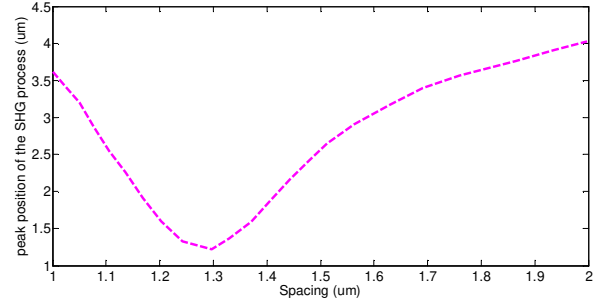

Figure 6. The optical power of the SHF peak and the position of the SHG process peak relative to the grating distance

In the next step, the grating depth and its duty cycle are also optimized. Figures 7 and 8 show the results of this optimization.
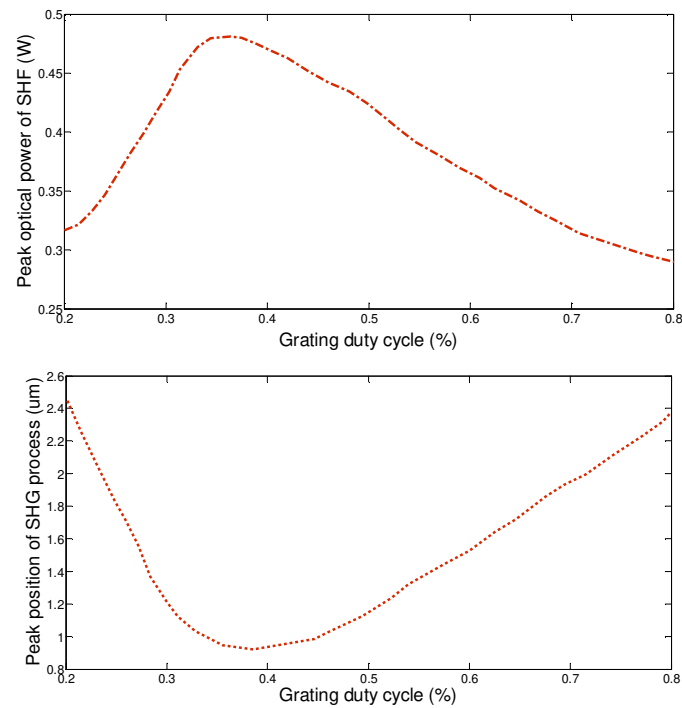

Figure 7. SHF peak optical power and position of the SHG peak relative to the duty cycle

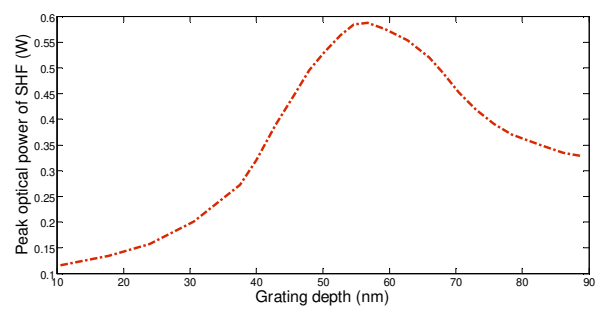




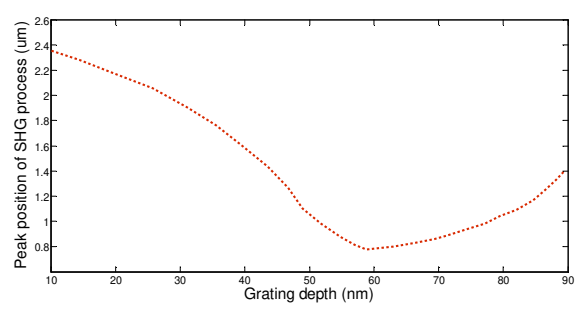

FIGURE 8. THE OPTICAL POWER OF THE SHF PEAK AND THE POSITION OF THE SHG PEAK RELATIVE TO THE GRATING DEPTH

So, the optimized proposed structure has the optical power SHG of $0.6 \mathrm{~W}$ and propagation length of $0.8 \mathrm{um}$. The results show that the proposed optimal structure has greatly improved the production of the second harmonic generation.

\section{Conclusions}

In this paper, we propose a new and optimal structure for the plasmonic waveguide so that the nonlinear optical effect of the second harmonic production is greatly improved. The proposed structure consists of two layers of metal at the top and bottom of the structure and a crystal layer of lithium niobytes (LiNbO3). A grating graphene layer is also added between the top metal and the crystal layer. The grate graphene layer leads to more absorption of photons and increases the absorption coefficient in the middle layer, thus increasing the production of the second harmonic. In addition, a number of spherical and elliptical nanoparticles of gold or silver have been added in the center of the layer. As will be shown, these nanoparticles increase the intensity of the optical field and thus increase the second harmonic effect. The number, shape (spherical or elliptical), and the material (gold or silver) of nanoparticle would be optimized to reach the maximum possible of SHG.

\section{Required declaration}

*Funding

There is no funding resources regarding the manuscript

*Conflicts of interest/Competing interests

The authors declare that they have no competing interests or conflict of interest

*Availability of data and material Not applicable

*Code availability

Not applicable'

*Authors' contributions
All authors contributed to the final version of the manuscript.

*Ethics approval

Not applicable

*Consent to participate

Not applicable

*Consent for publication

\section{NOT APPLICABLE}

\section{REFERENCES}

1. V. J. Sorger, R. F. Oulton, R.-M. Ma, and X. Zhang, "Toward integrated plasmonic circuits," MRS Bull., Vol. pp. 37(08), 728-738, 2012.

2. M. Yazdanypoora, and F. Emami, "Optimizing of the novel asymmetric plasmonic waveguide with two identical gratings to increase the SHG efficiency," Opt Quant Electron, 48:483, 2016.

3. R. F. Oulton, V. J. Sorger, D. A. Genov, D. F. P. Pile, and X. Zhang, "A hybrid plasmonic waveguide for sub-wavelength confinement and long-range propagation," Nat. Photonics, Vol. 2, pp. 496-500, 2008.

4. Jihua Zhang, Eric Cassan, Dingshan Gao, and Xinliang Zhang, "Highly efficient phase-matched second harmonic generation using an asymmetric plasmonic slot waveguide configuration in hybrid polymersilicon photonics" Optical Society of America, Vol. 21, 2013.

5. S.A. Maier, Plasmonics, Fundamentals and Applications, New York: Springer, 2007.

6. E. Ozbay, "Plasmonics: Merging photonics and electronics at nanoscale dimensions," Science, Vol. 311(5758), pp. 189-193, 2006.

7. R. X. Yang, R. A. Wahsheh, Z. L. Lu, and M. A. G. Abushagur, "Efficient light coupling between dielectric slot waveguide and plasmonic slot waveguide," Opt. Lett. Vol. 35, pp. 649-651, 2010.

8. P. Neutens, P. Van Dorpe, I. De Vlaminck, L. Lagae, and G. Borghs, "Electrical detection of confined gap plas-mons in Metal-Graphene-insulator-Metal waveguides," Nat. Photonics, Vol. 3, pp. 283-286, 2009.

9. J. Park, K. Y. Kim, I. M. Lee, H. Na, S. Y. Lee, and B. Lee, "Trapping light in plasmonic waveguides," Opt. Express, Vol. 18, pp. 598-623, 2010.

10. S. I. Bozhevolnyi, V. S. Volkov, E. Devaux, J. Y. Laluet, and T. W. Ebbesen, "Channel plasmon subwavelength waveguide components including interferometers and ring resonators," Nature, Vol. 440, pp. 508-511, 2006. 
11. J. Gosciniak, T. Holmgaard, and S. I. Bozhevolnyi, "Theoretical analysis of long-range dielectric-loaded surface plasmon polariton waveguides," J. Lightwave Technol. Vol. 29, pp. 1473-1481, 2011.

12. R. F. Oulton, V. J. Sorger, D. A. Genov, D. F. P. Pile, and X. Zhang, "A hybrid plasmonic waveguide for sub-wavelength confinement and long-range propagation," Nat. Photonics, Vol. 2, pp. 496-500, 2008.

13. J. A. Dionne, L. A. Sweatlock, and H. A. Atwater, "Plasmon slot waveguides: Towards chip-scale propagation with subwavelength-scale localization," Physical Review B, Vol. 73, pp. 035407, 2006.

14. R. Zia, M. D. Selker, P. B. Catrysse, and M. L. Brongersma, "Geometries and materials for subwavelength surface plasmon modes," Journal of the Optical Society of America A, Vol. 21, pp. 24422446, 2004.

15. Wen, K., Hu, Y., Chen, L., Zhou, J., Lei, L., \& Meng, Z. (2016). Single/dual Fano resonance based on plasmonic metal-dielectric-metal waveguide. Plasmonics, 11(1), 315-321.

16. Wen, K., Hu, Y., Chen, L., Zhou, J., He, M., Lei, L., \& Meng, Z. (2015). Multiple Plasmon-Induced Transparency Responses in a Subwavelength Inclined Ring Resonators System. IEEE Photonics Journal, 7(6), 1-7.

17. Wen, K., Hu, Y., Chen, L., Zhou, J., Lei, L., \& Guo, Z. (2015). Fano resonance with ultra-high figure of merits based on plasmonic Metal-Graphene-insulatorMetal waveguide. Plasmonics, 10(1), 27-32.

18. H. T. Miyazaki and Y. Kurokawa, "Squeezing visible light waves into a 3-nm-thick and 55-nm-long plasmon cavity," Physical Review Letters, Vol. 96, pp. 097401, 2006.

19. Y. Kurokawa and H. T. Miyazaki, "Metal-Grapheneinsulator-Metal plasmon nanocavities: Analysis of optical properties," Physical Review B, Vol. 75, 035411, 2007.

20. G. Veronis and S. Fan, "Bends and splitters in metaldielectric-metal subwavelength plasmonic waveguides," Applied Physics Letters, Vol. 87, 131102, 2005.

21. Y. Xie, Y. Huang, H. Che, W. Zhao, W. Xu, X. Li, and $\mathrm{J} . \mathrm{Li}$, "Theoretical investigation of a plasmonic sensor based on a metal-insulator-metal waveguide with a side-coupled nanodisk resonator" Journal of Nanophotonics, Vol. 9, Issue 1, 2015.

22. Y. Xie, Y. Huang, and W. Zhao, "A Novel Plasmonic Sensor Based on Metal-Insulator-Metal Waveguide With Side-Coupled Hexagonal Cavity" IEEE Photonics Journal, Vol. 7, Issue 2, 2015.

23. C. Zeng $\mathrm{n}$, and , Y. Cui, "Rainbow trapping of surface plasmon polariton waves in metal-insula tor-metal graded grating waveguide" Optics Communications Vol. 290, pp. 188 -191, 2013.

24. C. Zeng n, and , Y. Cui, "Low-disto rtion plasmonic slow- light system at telecommun ication regi me"
Optics Communications Vol. 294, pp. $372-376$, 2013.

25. R. W. Boyd, Nonlinear Optics, Academic, 2008.

26. T. Harimoto, B. Yo, K. Uchida, A novel multipass scheme for enhancement of second harmonic generation, Opt. Express, Vol. 19, 2011

27. H.J. Simon, D.E. Mitchell, J.G. Watson, "Optical second harmonic generation with surface plasmons in silver films", Phys. Rev. Lett., Vol. 33, 1974.

28. C.C. Neacsu, G.A. Reider, M.B. Raschke, "Second harmonic generation from nanoscopic metal tips: symmetry selection rules for single asymmetric nanostructures, Phys. Rev. B, Vol. 71, 2005.

29. C.G. Biris, N.C. Panoiu, "Second harmonic generation in metamaterials based on homogeneous centrosymmetric nanowires", Phys. Rev. B, Vol. 81(19), 2010.

30. F.F. Lu, T. Li, X.P. Hu, Q.Q. Cheng, S.N. Zhu, Y.Y. Zhu, "Efficient second harmonic generation in nonlinear plasmonic waveguide", Opt. Lett., Vol. 36, 2011.

31. S . Park, J.W. Hahn, J . Yong Lee, "Doubly resonant metallic nanostructure for high conversion efficiency of second harmonic generation", Opt. Express, Vol. $20,2012$.

32. S.B. Hasan, C. Rockstuhl, T. Pertsch, F. Lederer, "Second-order nonlinear frequency conversion processes in plasmonic slot waveguides", J. Opt. Soc. Am. B, Vol. 29, 2012.

33. M. Cazzanelli, F. Bianco, E. Borga, G. Pucker, M. Ghulinyan, E. Degoli, E. Luppi, V.Véniard, S. Ossicini, D. Modotto, S. Wabnitz, R. Pierobon, and L. Pavesi, "Second-harmonic generation in silicon waveguides strained by silicon nitride," Nat. Mater., Vol. 11(2), pp. 148-154, 2011.

34. J. S. Levy, M. A. Foster, A. L. Gaeta, and M. Lipson, "Harmonic generation in silicon nitride ring resonators," Opt. Express, Vol. 19(12), pp. 11415$11421,2011$.

35. R. E. P. de Oliveira, M. Lipson, and C. J. S. de Matos, "Electrically controlled silicon nitride ring resonator for quasi-phase matched second-harmonic generation," in CLEO: Science and Innovations, Optical Society of America, 2012.

36. T. Y. Ning, H. Pietarinen, O. Hyvärinen, R. Kumar, T. Kaplas, M. Kauranen, and G. Genty, "Efficient secondharmonic generation in silicon nitride resonant waveguide gratings," Opt. Lett. Vol. 37(20), pp. 4269-4271, 2012.

37. M. L. Brongersma and P. G. Kik, Surface Plasmon Nanophotonics, Springer, 2007.

38. M. I. Stockman, "Nanoplasmonics: past, present, and glimpse into future," Opt. Express, Vol. 19(22), pp. 22029-22106, 2011.

39. W. S. Cai, A. P. Vasudev, and M. L. Brongersma, "Electrically controlled nonlinear generation of light with plasmonics," Science, Vol. 333(6050), pp. 1720-1723, 2011. 
40. A. R. Davoyan, I. V. Shadrivov, and Y. S. Kivshar, "Quadratic phase matching in nonlinear plasmonic nanoscale waveguides," Opt. Express, Vol. 17(22), pp. 20063-20068, 2009.

41. S. B. Hasan, C. Rockstuhl, T. Pertsch, and F. Lederer, "Second-order nonlinear frequency conversion processes in plasmonic slot waveguides," J. Opt. Soc. Am. B, Vol. 29(7), pp. 1606-1611, 2012.

42. A. Ashkin, G. D. Boyd, J. M. Dziedzic, R. G. Smith, A. A. Ballman, J. J. Levinstein, and K. Nassau, "Optically-induced refractive index inhomogeneities in LiNbO3 and LiTaO3," Applied Physics Letters, Vol. 9(1), pp. 72-74, 1966.

43. A. Yariv, "Phase conjugate optics and real-time holography," IEEE Journal of Quantum Electronics, Vol. 14(9), pp. 650-660, 1978. 


\section{Figures}
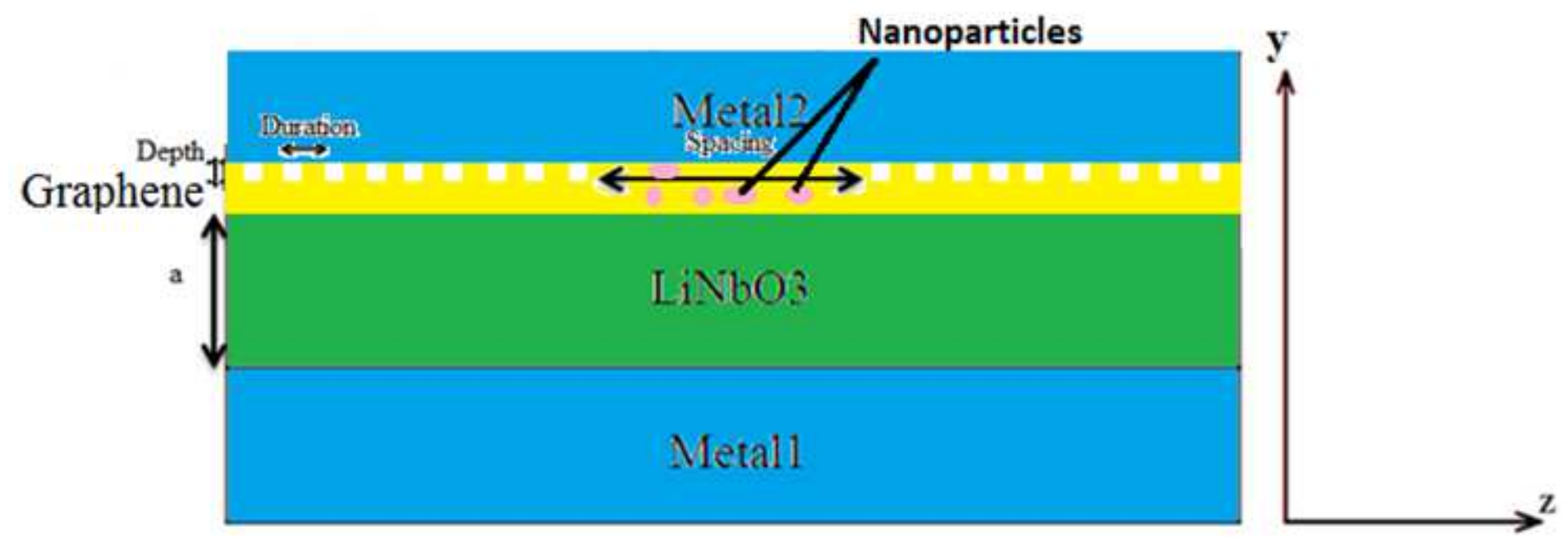

Figure 1

Structure of the proposed GRATING GRAPHENE MGIM waveguide under consideration
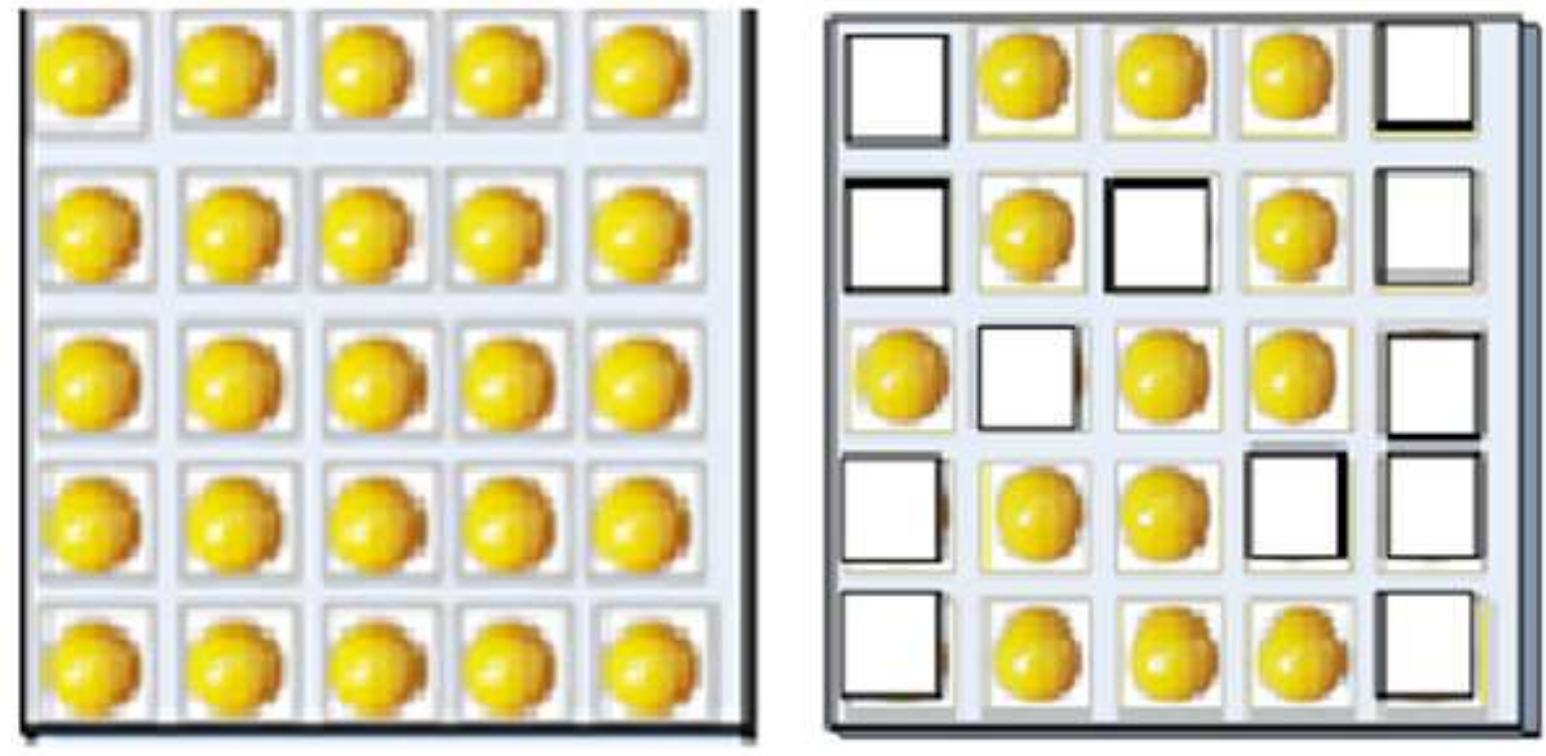

Figure 2

an alternating and non-alternating structure of nano-particles. 


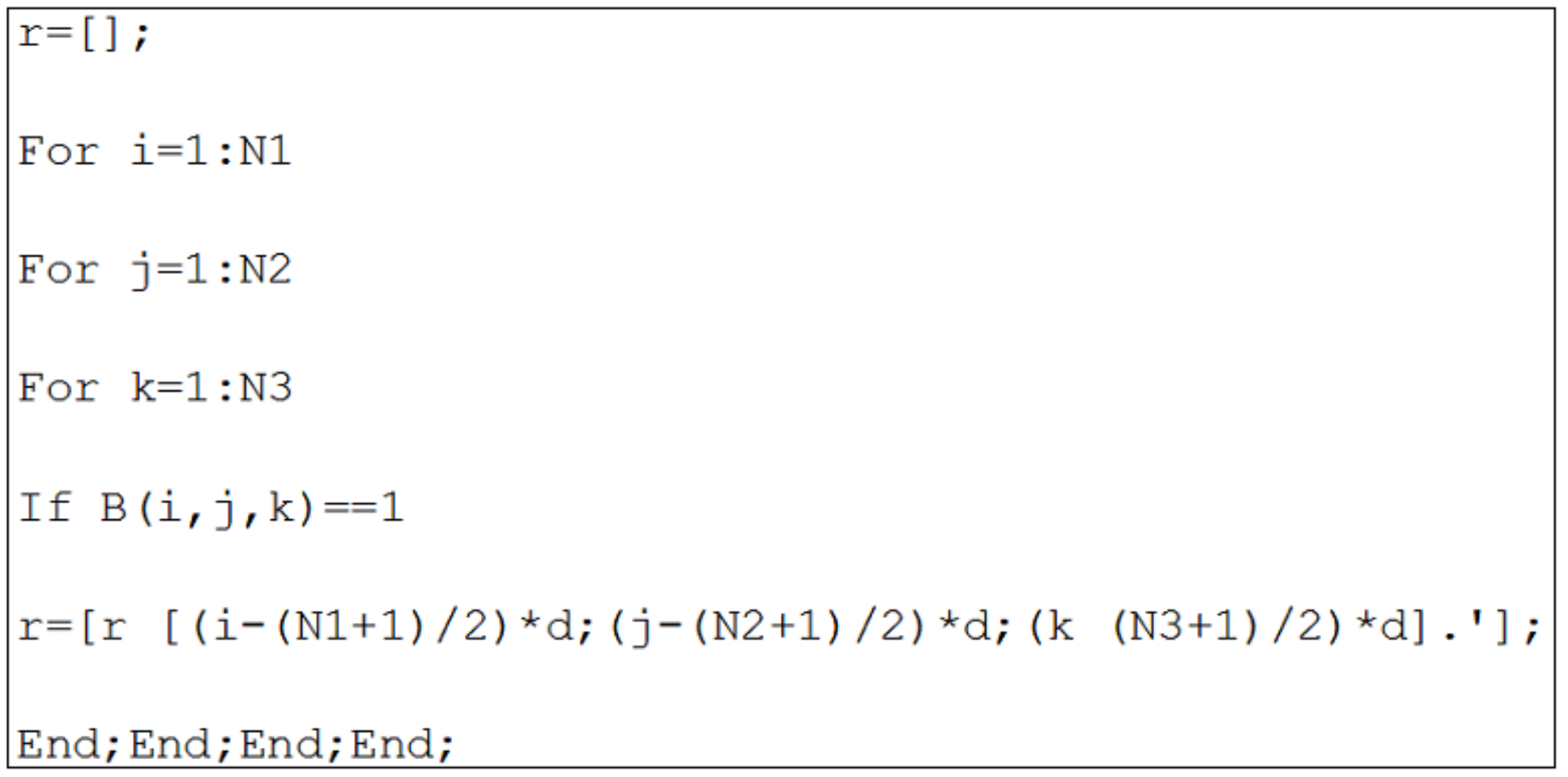

\section{Figure 3}

Nanoparticle positioning algorithm
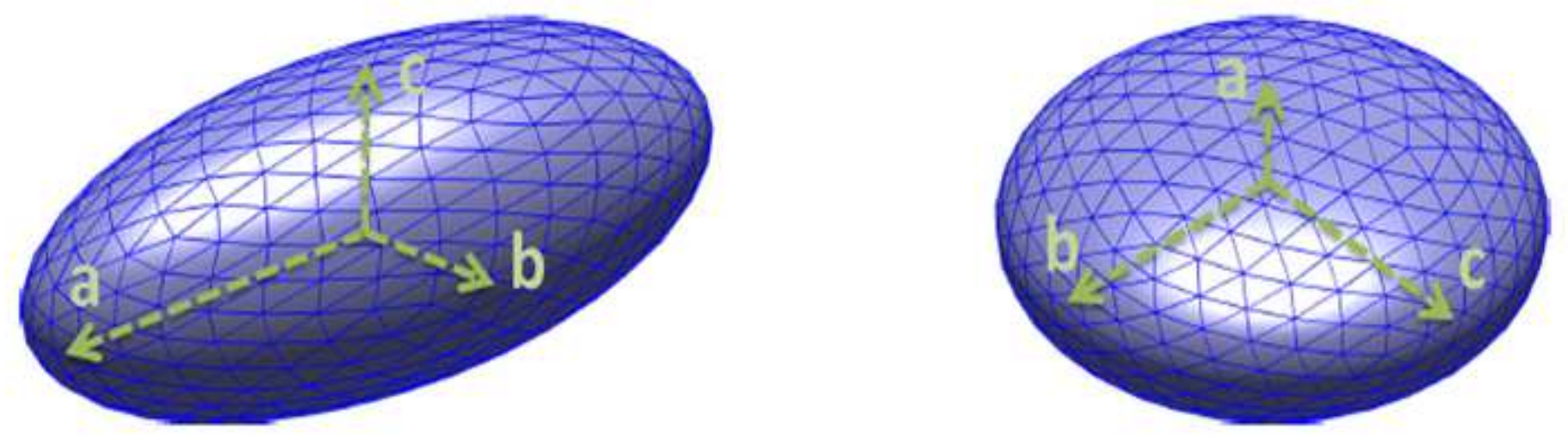

Figure 4

nanoparticles with different values of a, b, c 

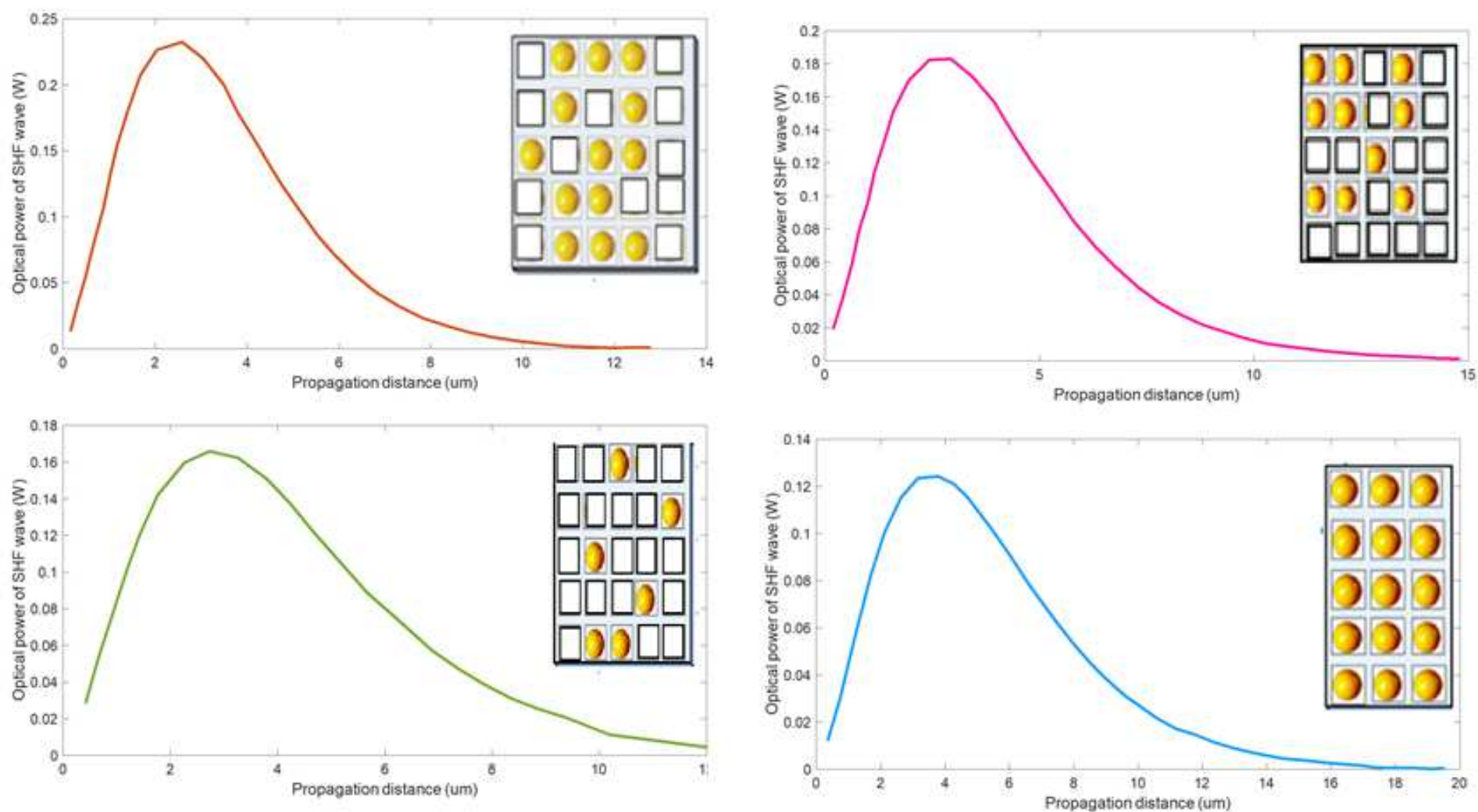

Figure 5

Comparison of SHG for periodic and nonperiodic structures

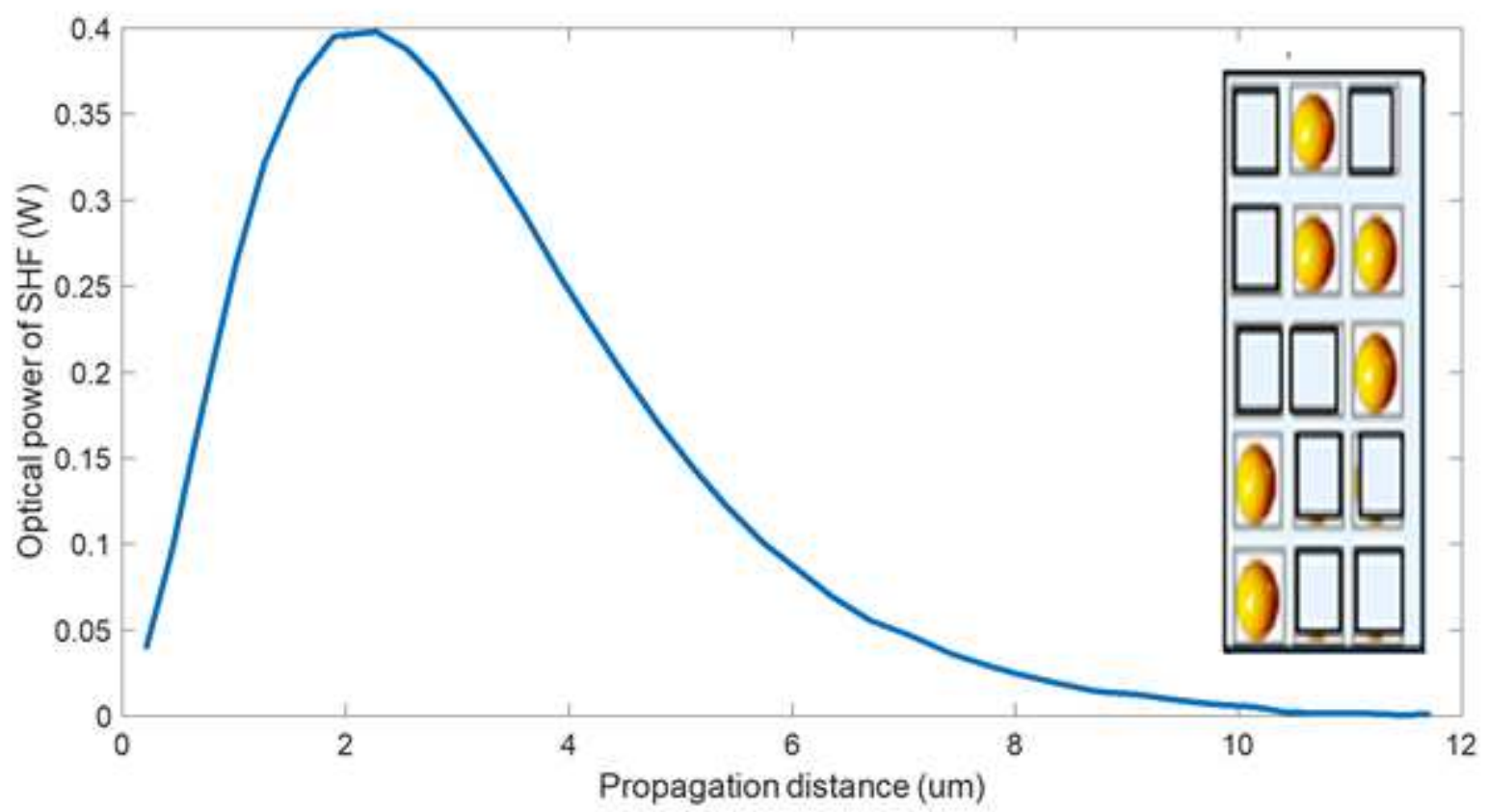

Figure 6

the optical power of SHG for proposed structure with optimized nanoparticles 

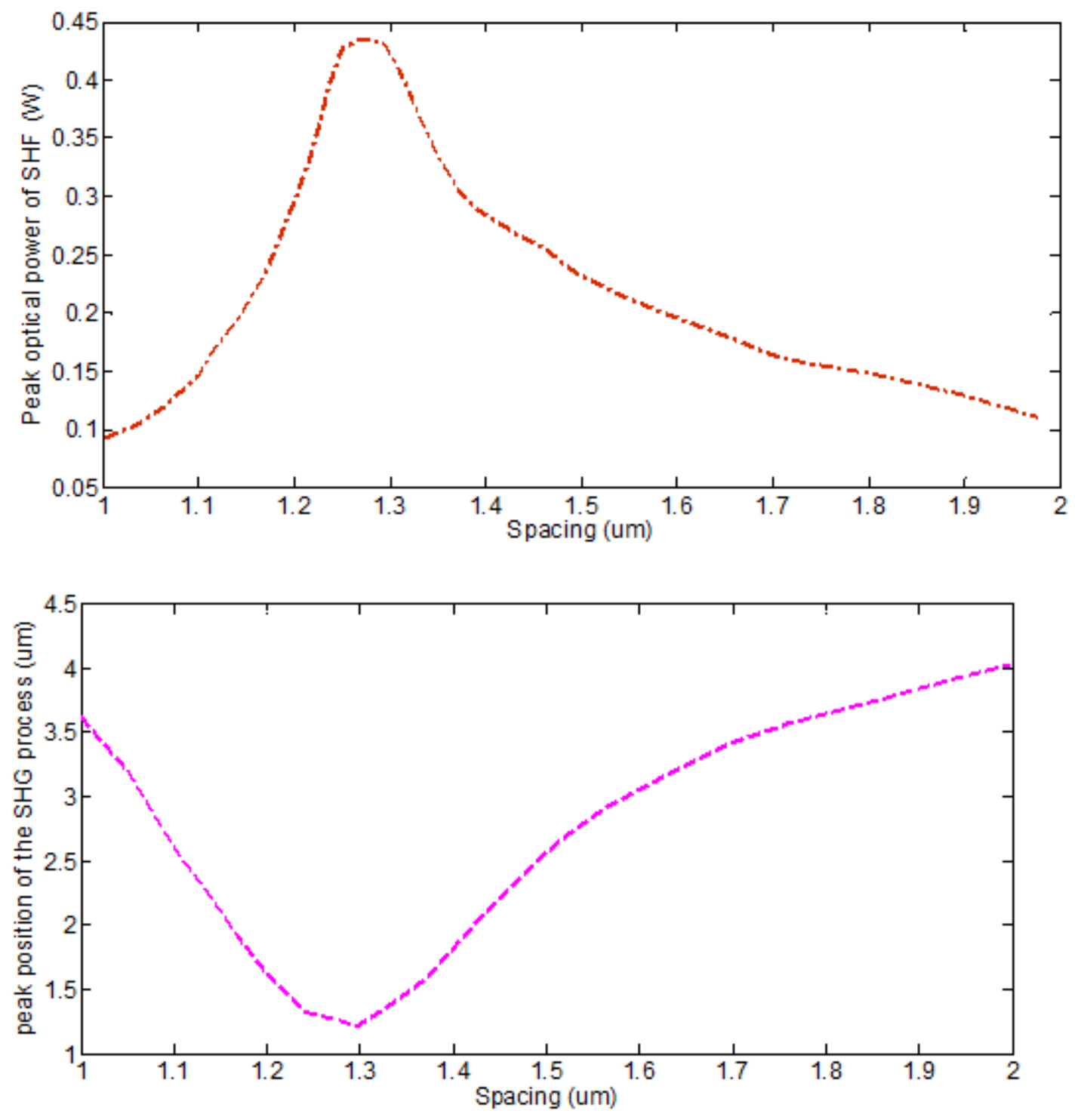

Figure 7

The optical power of the SHF peak and the position of the SHG process peak relative to the grating distance 

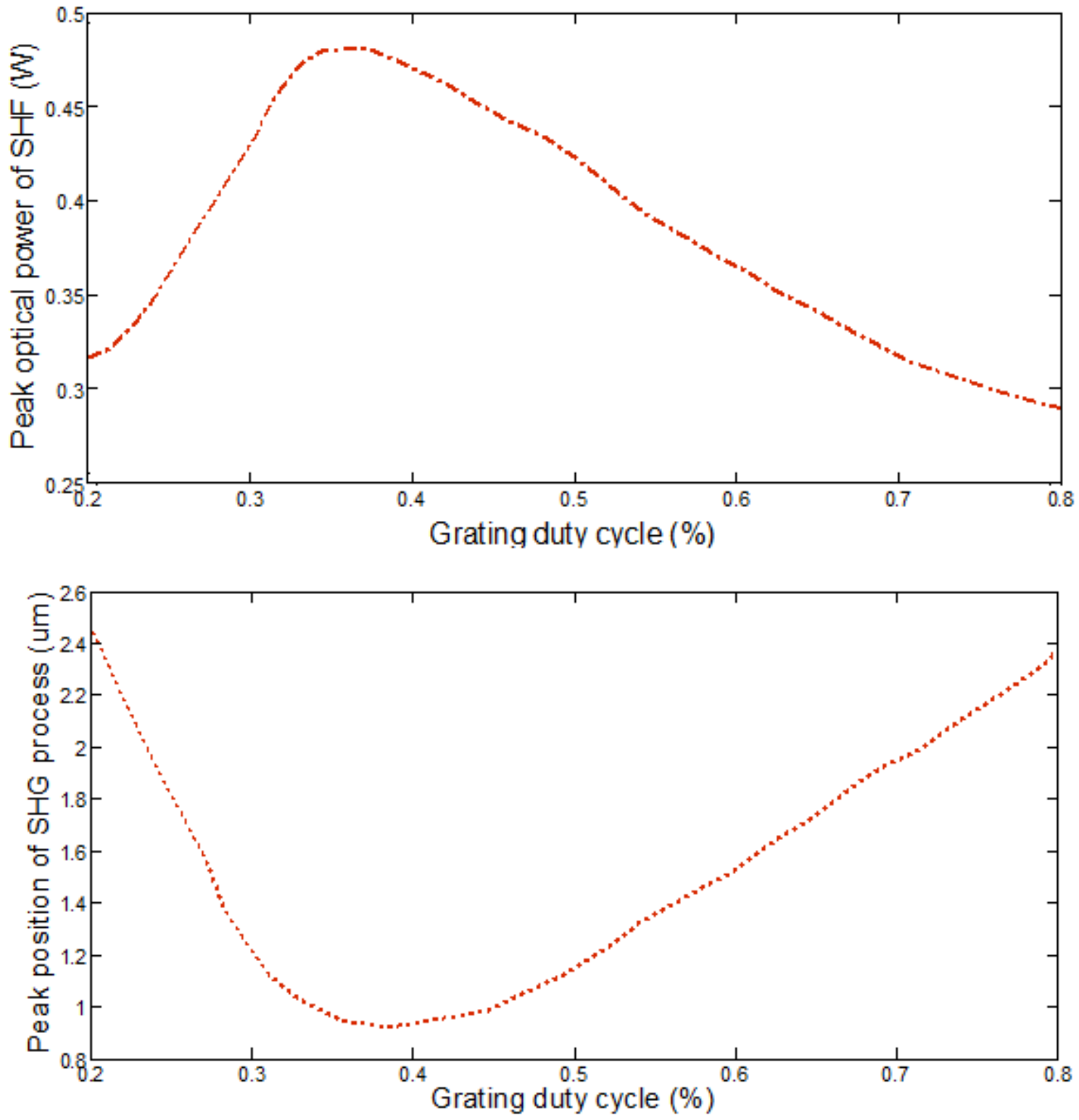

Figure 8

SHF peak optical power and position of the SHG peak relative to the duty cycle 

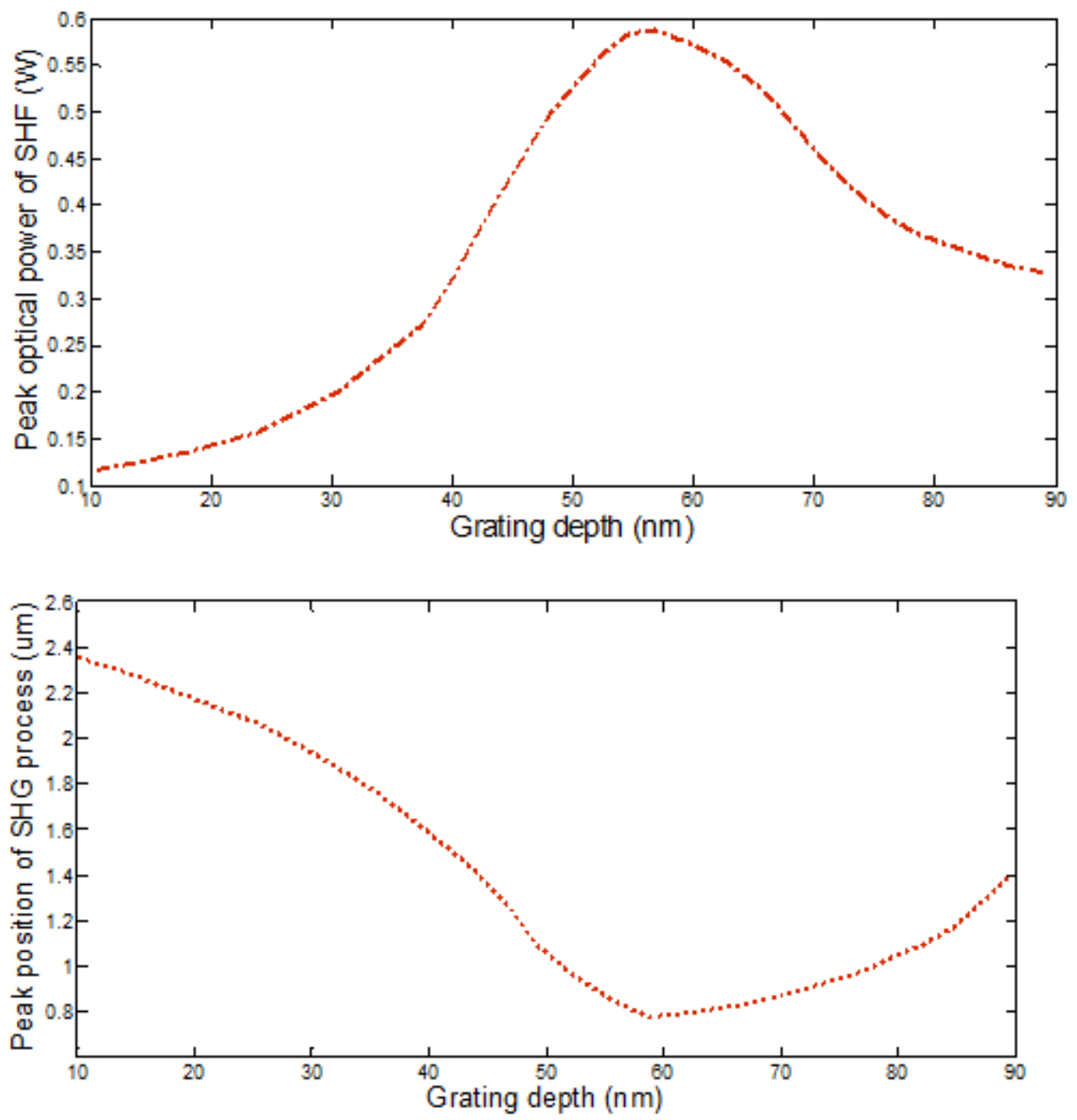

Figure 9

The optical power of the SHF peak and the position of the SHG peak relative to the grating depth 